\title{
Current Status of Awareness and Implementation of the "Yakuzai-Kanri Summary" in Community Pharmacies Prescribing Drugs under the Health Insurance System in Japan
}

\author{
Kazuhiro Watanabe ${ }^{* 1}$, Miyuku Chisima ${ }^{2}$, Kazuhiro Agata ${ }^{2}$ and Tomoyuki Hamamoto ${ }^{1}$ \\ Educational and Research Center for Clinical Pharmacy, Showa Pharmaceutical University ${ }^{1}$ Qol Co., Ltd. ${ }^{2}$ \\ $\left[\begin{array}{l}\text { Received December 19, } 2012 \\ \text { Accepted March 19, } 2013\end{array}\right]$
}

We investigated the awareness of the "Yakuzai-kanri Summary" (a pharmaceutical management summary) at community pharmacies in Japan that were prescribing drugs under the health insurance system. In addition, we assessed the pharmacy needs regarding receipt of the Summary. A cross-sectional survey was conducted by self-administered questionnaire between December 8 and December 30, 2011. Subjects were supervising pharmacists at 217 community pharmacies belonging to the Qol Group. The questionnaire comprised questions on the following: 1) awareness of the Yakuzai-kanri Summary; 2) Summary receipt rate; 3) pharmacy needs regarding receipt of the Summary; and 4) pharmacy needs regarding information on patient pharmaceutical care. Among the 178 community pharmacies that returned the self-administered questionnaire (response rate, 82.0\%), 73 (41.0\%) knew about the Yakuzai-kanri Summary and 6 (3.4\%) had previously received it. A total of 155 community pharmacies $(87.1 \%)$ were willing to receive the Yakuzai-kanri Summary to gain information on patient pharmaceutical care, especially information relating to discharge prescriptions, diagnosis, and treatment strategy. The results showed that the receipt rate of the Yakuzai-kanri Summary remains low, although community pharmacies are keen to receive it.

\footnotetext{
Key words — community pharmacies, health insurance system, the Yakuzai-kanri Summary, collaboration between community and hospital pharmacies
}

\section{Introduction}

The coordinated medical system that provides continuous and consistent medical care for inpatient, outpatient, and home-care treatment in Japan has been attracting attention in recent years. Doctors in private practice and hospital-employed physicians share patient medical information by exchanging referral documents. Similarly, hospital-employed nurses and visiting nurses share patient information by means of summarizing nursing reports. Hospital pharmacies and community pharmacies in Japan prescribing drugs under the health insurance system (community pharmacies) share information on drugs prescribed to patients through the personal medication record booklet. To date however, there is no means of sharing information on pharmaceutical care of individual patients while hospitalized. Following several reports highlighting the need for providing information on patient pharmaceutical care to community pharmacists by hospital pharmacists, ${ }^{1,2)}$ the Japanese Society of Hospital Pharmacists started encouraging society members in October 2005 to issue the "Yakuzai-kanri Summary" (a pharmaceutical management summary), which contains information on pharmaceutical care provided during inpatient treatment (Fig. 1). ${ }^{3-5)}$ In April 2008,

* 3-3165, Higashi-Tamagawagakuen, Machida-shi, Tokyo, 194-8543 Japan 


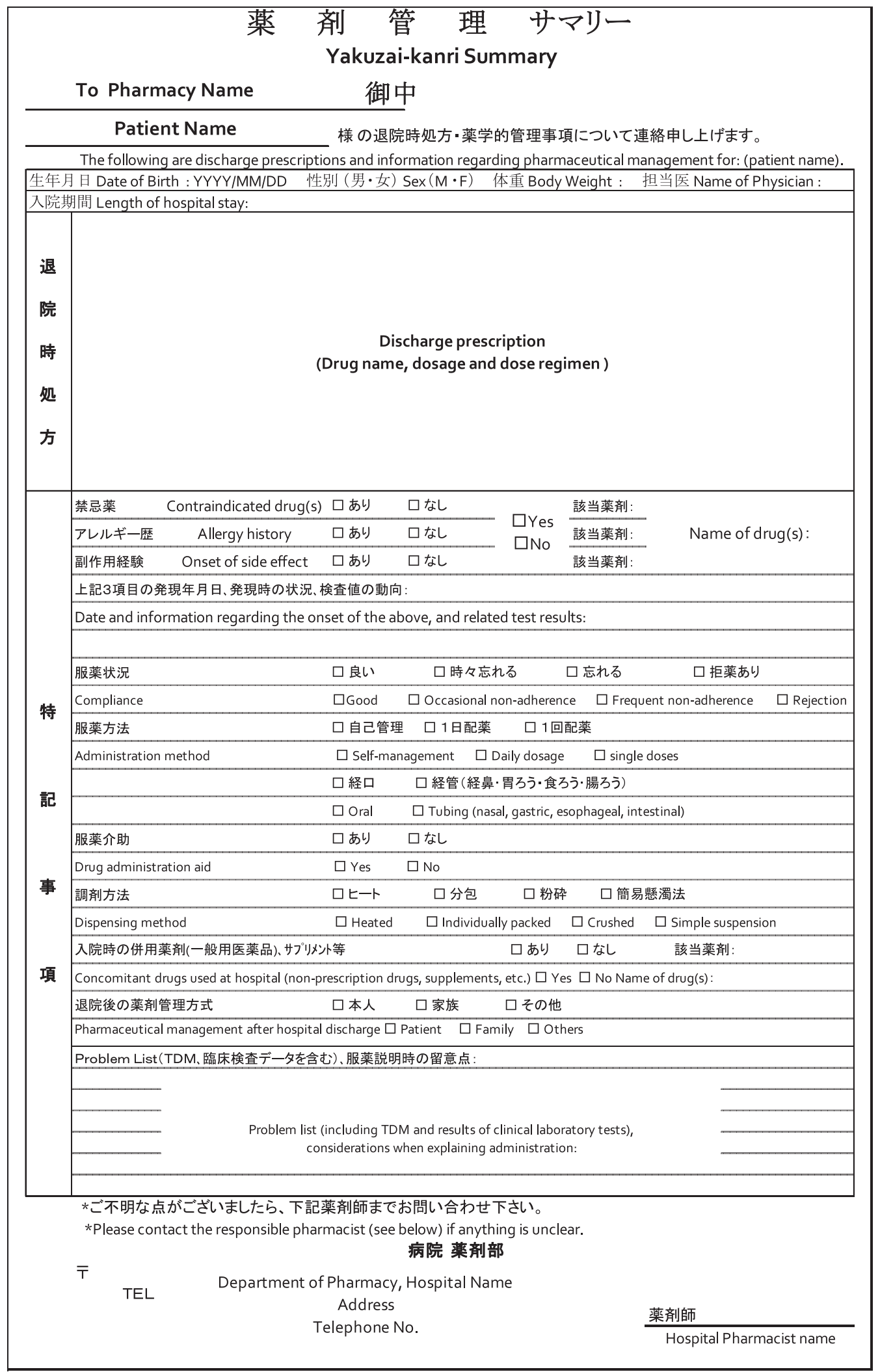

Fig. 1 Sample of "Yakuzai-kanri Summary"

such information sharing was recognized as a component of the national health insurance system: the costs for the "pharmaceutical informa- tion management and counseling at hospital discharge" service, which involves the provision of documents containing pharmaceutical care infor- 
mation to medical institutions and community pharmacies, became a claimable component of medical treatment fees. ${ }^{5,6}$

In this study, we aimed to gain insight into the current status (as of 2011) of awareness and on the implementation of the Yakuzai-kanri Summary by community pharmacies in Japan. We also investigated the needs of community pharmacies regarding receipt of the Summary, and we discuss how the Summary should work within the collaboration framework between hospital and community pharmacies.

\section{Methods}

\section{Study population}

A cross-sectional survey was conducted by self-administered questionnaire. The questionnaire was sent to supervising pharmacists at 217 community pharmacies belonging to the Qol Group across 31 prefectures in Japan. All pharmacies dispensed prescribed drugs. Before conducting the survey, the aim and purpose of the study were explained by e-mail to the potential participants, and those who agreed to participate in the study received a sample of the Yakuzaikanri Summary (Fig. 1) prepared by the Japanese Society of Hospital Pharmacists and a written explanation of the system regarding "pharmaceutical information management at hospital discharge”. The survey period was December 12-30, 2011. Questionnaire items were as follows.

\section{Research Instruments}

\section{Awareness of the Yakuzai-kanri Sum- mary}

Question: Do you know about the Yakuzai-kanri Summary?
Response options: “yes" and "no".

Data were analyzed after stratification according to the characteristics of the community pharmacies that responded to the survey to identify variables influencing the awareness of the Yakuzaikanri Summary.

\section{Receipt of the Yakuzai-kanri Summary}

Question: Has your pharmacy ever received the Yakuzai-kanri Summary, or "discharge instructions addressing medication" that includes pharmaceutical information for inpatient treatment and/or the personal medication record booklet?

Response options: 1) Our pharmacy has received the Yakuzai-kanri Summary; 2) Our pharmacy has received "discharge instructions addressing medication" and/or the personal medication record booklet; and 3) Our pharmacy has never received such information.

Community pharmacies that responded to the survey were categorized as follows: those that received the Yakuzai-kanri Summary (those who selected option 1 or option 2) or those that did not receive the summary (those who selected option 3). Data were analyzed after stratification according to the characteristics of the community pharmacies that responded to the survey to identify variables influencing the receipt of the Yakuzaikanri Summary.

\section{Needs regarding the receipt of the Yakuzai-kanri Summary}

Question: How keen or hesitant are you to receive the Yakuzai-kanri Summary?

Response options were in the form of a 5-point Likert scale $(1=$ very keen, $2=$ keen, $3=$ neither keen nor hesitant, $4=$ hesitant, and $5=$ very hesitant). 


\section{Needs regarding patient pharmaceuti- cal care}

\section{4-1. Important items in the Yakuzai-kanri Summary}

Question: Which of the Yakuzai-kanri Summary items do you think is particularly important for community pharmacists? Please select the three most important options from the following ten options.

Response options: discharge prescriptions; contraindicated drugs and allergy history; previously experienced side effects; compliance; administration methods (including the simple suspension method); drug administration aid; dispensing method; concomitant drugs used during inpatient treatment (non-prescription drugs and supplements); person(s) responsible for pharmaceutical management after hospital discharge (patient, family or carer); and problems in pharmaceutical care during inpatient treatment prepared by a hospital pharmacist.

\section{4-2. Important items currently not included in the}

\section{Yakuzai-kanri Summary}

Question: Which items currently not included in the Yakuzai-kanri Summary do you consider important for community pharmacists? Please select the three most important options from the following nine options.

Response options: medication changes and withdrawal during inpatient treatment and associated reasons; information on diagnosis and treatment strategy; disease notification status; special information for high-risk drugs (pharmacotherapy regimen, wash-out period, symptoms of side effects that needs to be monitored); purpose of offlabel use; instructions for necessary maneuvers for medication; description of administration; patient ability to manage medication, including compliance, and activities of daily living.

\section{4-3. Important information regarding diseases}

Question: Select the three most important diseases from the list of eight for which you would like to acquire pharmaceutical care information.

Response options: cancer; psychiatric disorders; diabetes mellitus; diseases approved by the home medical care system; cardiovascular diseases; pediatric diseases; respiratory diseases and infectious diseases; other (please describe).

The characteristics of respondents (supervising pharmacists) and their workplaces (community pharmacies) were also examined.

\section{Statistical analysis}

Data on the awareness and receipt of the Yakuzai-kanri Summary, after stratification, were analyzed using the Chi-square test for statistical significance (two-way, $P<0.005$ ) to identify the variables influencing survey outcomes. IBM SPSS Statistics 20.0 was used for statistical analysis.

\section{Ethical approval}

The study protocol was designed to adhere to the ethical guidelines for epidemiologic studies and was approved by the research ethics committee of Showa Pharmaceutical University (Approval number $\mathrm{H} 23-12)$.

\section{Results}

\section{Study population}

A total of 178 pharmacies across 30 prefectures belonging to the Qol Group returned the self-administered questionnaire (valid response rate, $82.0 \%)$. The characteristics of the respondents (supervising pharmacists) and their workplaces (community pharmacies) are shown in Table $\mathbf{1}$. 
Table 1 The characteristics of the respondents and their workplaces

\begin{tabular}{|c|c|c|}
\hline & Variable & All $(n=178)$ \\
\hline \multicolumn{3}{|c|}{ Characteristics of respondents (supervising pharmacists) } \\
\hline Sex & Female - no. $(\%)$ & $101(56.7 \%)$ \\
\hline \multirow[t]{5}{*}{ Age } & $20-29-$ no. $(\%)$ & $17(9.6 \%)$ \\
\hline & $30-39-$ no. $(\%)$ & $37(20.8 \%)$ \\
\hline & $40-49-$ no. $(\%)$ & $53(29.8 \%)$ \\
\hline & $50-59-$ no. $(\%)$ & $54(30.3 \%)$ \\
\hline & $60-69-$ no. $(\%)$ & $17(9.6 \%)$ \\
\hline \multicolumn{2}{|r|}{ with previous experience as a hospital pharmacist - no. (\%) } & $30(16.8 \%)$ \\
\hline \multicolumn{3}{|c|}{ Characteristics of respondent workplaces (community pharmacies) } \\
\hline \multicolumn{2}{|r|}{ Median number of pharmacists - no. (range) } & $4(1-14)$ \\
\hline \multicolumn{2}{|r|}{ Median number of monthly prescriptions filled - no. (range) } & $1,750(30-6,000)$ \\
\hline \multicolumn{2}{|r|}{ Median number of monthly prescription filled per pharmacist - no. (range) } & $600(15-1,700)$ \\
\hline \multicolumn{2}{|r|}{$\begin{array}{l}\text { pharmacies per estimated number of total prescriptions issued by physicians } \\
\text { - \% (range) }\end{array}$} & $60.0(1-100)$ \\
\hline \multicolumn{2}{|c|}{ Median Bungyo ratio (regional) — \% (range) } & $66.7(33.3-79.8)$ \\
\hline \multicolumn{2}{|r|}{$\begin{array}{l}\text { Number of pharmacies participating in collaboration between hospital and } \\
\text { community pharmacies, such as seminars and meetings for information exchange } \\
\text { - no. (\%) }\end{array}$} & $71(39.9 \%)$ \\
\hline
\end{tabular}

\section{Research Instruments}

\section{Awareness of the Yakuzai-kanri Sum- mary}

A total of 73 pharmacies (41.0\%) knew about the Summary, while 105 (59.0\%) did not. Data were analyzed after stratification according to the characteristics of the community pharmacies that responded to the survey (Table 2). Results revealed that awareness of the Yakuzai-kanri Summary was significantly higher in community pharmacies collaborating with hospital pharmacies than in those not collaborating $(P=0.04)$. No other stratification variables were statistically significant.

\section{Receipt of the Yakuzai-kanri Summary}

Six community pharmacies (3.4\%) had previously received the Summary and 8 (4.5\%) had received the "discharge instructions addressing medication" that include pharmaceutical information for inpatient treatment and/or the personal medication record booklet. On the other hand, 164 pharmacies $(92.1 \%)$ had never received any of the above documents.

Data were further analyzed after stratification according to community community pharmacies (Table 2). Results revealed that receipt of the Yakuzai-kanri Summary was significantly higher in community pharmacies collaborating with hospital pharmacies than in those not collaborating $(P$ $<0.01)$. No other stratification variables were statistically significant.

\section{Needs regarding receipt of the Yakuzai- kanri Summary}

A total of 115 pharmacies (87.1\%) were very keen $(n=64,36.0 \%)$ or keen $(n=91,51.1 \%)$ to receive the Summary. On the other hand, 13 pharmacies $(7.3 \%)$ were neither keen nor hesitant to receive it, $2(1.1 \%)$ were hesitant to receive it, 2 $(1.1 \%)$ were very hesitant to receive it, and 6 (3.4\%) gave no response.

\section{Needs regarding patient pharmaceuti- cal care}

4-1. Important items in the Yakuzai-kanri Summary

Table 3 shows the items of the Summary that 
Table 2 The stratified analysis on community pharmacies confounders

\begin{tabular}{|c|c|c|c|c|c|c|c|}
\hline \multirow[t]{2}{*}{ Factors: Characteristics of community pharmacies } & & \multicolumn{3}{|c|}{$\begin{array}{l}\text { Awareness of } \\
\text { the Yakuzai-kanri Summary }\end{array}$} & \multicolumn{3}{|c|}{$\begin{array}{l}\text { Receipt of } \\
\text { the Yakuzai-kanri Summary }\end{array}$} \\
\hline & & Yes & No & $P$ Value & Yes & No & $P$ Value \\
\hline \multirow{2}{*}{ Median number of pharmacists } & $4 \leq$ & 48 & 56 & \multirow{2}{*}{0.12} & 5 & 99 & \multirow{2}{*}{0.4} \\
\hline & $<4$ & 25 & 49 & & 1 & 73 & \\
\hline \multirow{2}{*}{ Median number of monthly prescriptions filled } & $1,750 \leq$ & 38 & 51 & \multirow{2}{*}{0.76} & 5 & 84 & \multirow{2}{*}{0.21} \\
\hline & $<1,750$ & 35 & 54 & & 1 & 88 & \\
\hline \multirow{2}{*}{ Median number of monthly prescription filled per pharmacist } & $600 \leq$ & 33 & 64 & \multirow{2}{*}{0.05} & 2 & 95 & \multirow{2}{*}{0.41} \\
\hline & $<600$ & 40 & 41 & & 4 & 77 & \\
\hline \multirow{2}{*}{$\begin{array}{l}\text { Median the mean percentage of prescription dispensed at the respondents' } \\
\text { pharmacies per estimated number of total prescriptions issued by physicians }\end{array}$} & $60.0 \% \leq$ & 35 & 56 & \multirow{2}{*}{0.55} & 3 & 88 & \multirow{2}{*}{1} \\
\hline & $<60.0 \%$ & 37 & 49 & & 3 & 83 & \\
\hline \multirow{2}{*}{ Median Bungyo ratio (regional) } & $66.7 \% \leq$ & 43 & 56 & \multirow{2}{*}{0.54} & 5 & 94 & \multirow{2}{*}{0.23} \\
\hline & $<66.7 \%$ & 30 & 49 & & 1 & 78 & \\
\hline \multirow{2}{*}{$\begin{array}{l}\text { Participation in collaboration between hospital and community } \\
\text { pharmacies, including seminars and meetings for information exchange }\end{array}$} & Yes & 36 & 35 & \multirow{2}{*}{$0.04 *$} & 6 & 65 & \multirow{2}{*}{$<0.01^{* *}$} \\
\hline & No & 37 & 70 & & 0 & 107 & \\
\hline
\end{tabular}

Table 3 Needs regarding patients' pharmaceutical care (1): Important items in the Yakuzai-kanri Summary

\begin{tabular}{|c|c|c|}
\hline \multirow{2}{*}{ Item } & \multicolumn{2}{|c|}{$\mathrm{n}=178$} \\
\hline & $\%$ & Number \\
\hline Discharge prescriptions & 72.5 & 129 \\
\hline Dispensing method & 71.3 & 127 \\
\hline Administration methods (including the simple suspension method) & 47.8 & 85 \\
\hline Contraindicated drugs and allergy history & 40.4 & 72 \\
\hline Previously experienced side effects & 39.3 & 70 \\
\hline Problems in pharmaceutical care during inpatient treatment prepared by a hospital pharmacist & 34.8 & 62 \\
\hline Person(s) responsible for pharmaceutical management after hospital discharge (patient, family or carer) & 23.0 & 41 \\
\hline Compliance & 20.8 & 37 \\
\hline Concomitant drugs used during inpatient treatment (non-prescription drugs and supplements) & 19.1 & 34 \\
\hline Drug administration aid & 12.9 & 23 \\
\hline
\end{tabular}

respondents regarded as most important. Most respondents required information on discharge prescriptions ( $\mathrm{n}=129 ; 72.5 \%)$, dispensing methods $(\mathrm{n}=127 ; 71.3 \%)$, and administration methods including the simple suspension method $(\mathrm{n}=85$; $47.8 \%)$.

\section{4-2. Important items not included in the Yakuzai-}

\section{kanri Summary}

Table 4 shows items that respondents regarded as important but that are not currently (as of 2011) provided in the Yakuzai-kanri Summary. Most respondents wanted information on diagnosis and treatment strategy $(\mathrm{n}=140 ; 78.7 \%)$, followed by disease notification status $(n=89$;
$50.0 \%)$, and purpose of off-label use $(\mathrm{n}=75$; $42.1 \%)$.

\section{4-3. Important information regarding diseases}

Table 5 shows the type of diseases for which respondents required information. Most respondents required disease information on cancer $(\mathrm{n}=$ $160 ; 89.9 \%$ ), followed by psychiatric disorders ( $\mathrm{n}$ $=110 ; 61.8 \%)$ and diabetes mellitus $(\mathrm{n}=100$; $56.2 \%)$.

\section{Discussion}

This study revealed the current status of awareness and implementation of the Yakuzai-kanri 
Table 4 Needs regarding patients' pharmaceutical care (2): Important items currently not included in the Yakuzai-kanri Summary

\begin{tabular}{|c|c|c|}
\hline \multirow{2}{*}{ Item } & \multicolumn{2}{|c|}{$\mathrm{n}=178$} \\
\hline & $\%$ & Number \\
\hline Information on diagnosis and treatment strategy & 78.7 & 140 \\
\hline Purpose of off-label use & 50.0 & 89 \\
\hline Disease notification status & 42.1 & 75 \\
\hline Medication changes and withdrawal during inpatient treatment and their reasons & 31.5 & 56 \\
\hline $\begin{array}{l}\text { Special information for high-risk drugs (pharmacotherapy regimen, wash-out period, } \\
\text { symptoms of side effects that needs to be monitored) }\end{array}$ & 11.2 & 20 \\
\hline Patient ability to manage medication, including compliance, and activities of daily living & 7.3 & 13 \\
\hline Description of administration & 4.5 & 8 \\
\hline Instructions for necessary maneuvers for medication & 3.9 & 7 \\
\hline
\end{tabular}

Table 5 Needs regarding patients' pharmaceutical care (3): Important information regarding diseases

\begin{tabular}{|c|c|c|}
\hline \multirow{2}{*}{ Item } & \multicolumn{2}{|c|}{$\mathrm{n}=178$} \\
\hline & $\%$ & Number \\
\hline Cancer & 89.9 & 160 \\
\hline Psychiatric disorders & 61.8 & 110 \\
\hline Diabetes mellitus & 56.2 & 100 \\
\hline Diseases approved by the home medical care system & 54.5 & 97 \\
\hline Cardiovascular diseases & 50.0 & 89 \\
\hline Pediatric diseases & 39.9 & 71 \\
\hline Respiratory diseases and infectious diseases & 38.8 & 69 \\
\hline Others & 3.9 & 7 \\
\hline
\end{tabular}

Summary in community pharmacies as well as their willingness to receive the Summary. We also identified specific elements of information required by community pharmacists on patient pharmaceutical care while hospitalized.

We found that only $41.0 \%$ of community pharmacies knew about the Yakuzai-kanri Summary, even though use of the Summary has been encouraged for 6 years and the system for "pharmaceutical information management at hospital discharge" has been in operation for 3 years. To improve this poor recognition of the Summary, both the Japanese Pharmaceutical Association and the Japanese Society of Hospital Pharmacists need to focus on further promotion and education.

In addition, analysis of data stratified according to the characteristics of the community pharma- cies that responded to the survey revealed that collaboration between community and hospital pharmacies is a significant stratification variable in the awareness of the Yakuzai-kanri Summary. This finding indicates that the collaboration influences the awareness of the Summary and suggests that promoting the collaboration between community and hospital pharmacies in each region is critical for increasing the awareness of the Yakuzai-kanri Summary.

We also found that a low percentage $(<10 \%)$ of community pharmacies had received any kind of document concerning patient pharmaceutical care: only $3.4 \%$ had received the Summary and $5.6 \%$ had received other documents such as "discharge instructions addressing pharmaceutical care during the inpatient treatment" and/or the 
personal medication record booklet. These findings are of particular importance since they were the first results reported on the receipt rate of the Summary in the community. The Japanese Society of Hospital Pharmacists conducted a nationwide survey on the provision of discharge instruction by hospital pharmacists in 2008 and found that $5.6 \%$ of respondents were previously issued the Yakuzai-kanri Summary. ${ }^{3)}$ Although it is difficult to directly correlate this result with the present results, the receipt rate of the Summary will not increase without improving such low issuance rates.

Analysis of the results of the receipt of the Summary after stratification according to the characteristics of the community pharmacies showed that collaboration between community and hospital pharmacies is also a significant stratification variable for the receipt of the Summary. This indicates that the collaboration influences the receipt of the Summary. It is possible that the receipt rate of the Yakuzai-kanri Summary in community pharmacies will increase if more pharmacies collaborate in the future.

In the present study, we showed that $87 \%$ of community pharmacies are willing to receive the Summary. However, it appears that hospital pharmacies are unable to issue the Summary to meet such high demand. With respect to the reasons hindering hospital pharmacists issuing the Yakuzai-Kanri Summary, a survey carried out by the Japanese Society of Hospital Pharmacists in $2008^{3)}$ on the status of the responsibilities of hospital pharmacists with regard to discharge instruction identified the following two main reasons. First, hospital pharmacists have difficulties in finding time to prepare the Summary (mentioned by $44.7 \%$ of respondents). Second, hospital pharmacists do not believe that the Summary is as necessary as drug information leaflets and that personal medication record booklets suffice to serve the purpose (mentioned by $42.4 \%$ of respondents). Underlying these problems, for example, whether or not the Summary has too many items to fill in and/or is presented in an unacceptable format, or whether or not everyday responsibilities of hospital pharmacists are hindering issuing of the Summary in the clinical setting, remain unclear.

Thus, we believe that establishing the conditions wherein hospital pharmacists can smoothly issue the Summary is essential. For this however, as our factor analysis indicates, collaboration between hospital and community pharmacies is crucial. More precisely, it is important to shape and implement collaboration that helps both hospital and community pharmacists understand each other, increase awareness of the benefit of using the Yakuzai-kanri Summary as a means to share patient information, and cooperatively address the underlying problems while finding solutions for increasing the Summary issue rate.

Taken together, we believe that establishing conditions that enable smooth issuing of the Summary by hospital pharmacists are essential and that collaboration and good understanding between hospital and community pharmacies will play a pivotal role in establishing such conditions. Both hospital and community pharmacists should recognize the benefit of using the Yakuzai-kanri Summary as a means to share patient information while cooperatively addressing the underlying problems and finding solutions for using the Summary in a practical manner. This study provides important insight into the detailed aspects of patient information on pharmaceutical care required by community pharmacists. In good agreement with the results of a previous study carried out by 
the Japanese Pharmaceutical Association in $2005,{ }^{3)}$ we found that a high percentage of community pharmacists are willing to receive information on the following aspects: discharge prescriptions, dispensing methods, and diagnosis and treatment strategy. Information on these aspects of pharmaceutical care is not included in prescriptions or in personal medication record booklets and is difficult to obtain directly from patients. Thus, revision of the Yakuzai-kanri Summary to focus on such highly desirable aspects of pharmaceutical care may address hospital pharmacist problems related to the Summary items. We also found that approximately $90 \%$ of community pharmacists were willing to receive information on pharmaceutical care for cancer patients. This suggests that the revision to reflect such needs will improve the utility of the Summary and consequently expand its usage.

Although this study is a nationwide survey, all respondents were from community pharmacies belonging to the Qol Group, and thus our results may only represent the status of recognition and receipt of the Yakuzai-kanri Summary in a subpopulation of community pharmacies. Nevertheless, this study highlights the latest status of the Summary in community pharmacies while providing insight into specific elements of information on pharmaceutical care required by community pharmacists. We believe that our findings will serve as a solid platform for reviewing the contents of Yakuzai-kanri Summary and the sharing patient information within the collaboration framework between hospital and community pharmacies.

\section{Acknowledgements}

This work was supported by Grant-in-Aid for Young Scientists of Showa Pharmaceutical University (No. 23-4). We greatly appreciate the cooperation of the community-based pharmacies involved in this study.

\section{References}

1) Edo K, Takahashi T, Yakuzaishi ga Okonau Taiin-ji no Kanjya Shido [Clinical pharmacy services to in patients at the time of hospital discharge], Pharmaceuticals Monthly, 2006, 48, 981-985.

2) Miyazaki Y, Yakuzaishi niyoru Chiiki-Renkei [The Role of the Pharmacist in the Community Health Care], Pharmaceuticals Monthly, 2009, 51, 27-33.

3) Sato H, Kase Y, Ando T, Okamura M, Okimura A, Sugiura T, Tanaka K, Tahara M, Natsume N, Miyazaki Y, Koinuma M, Taiin-ji Shidou-Gyoumu ni Kansuru Genjyo-Houkoku [Status report on Discharge instruction of Hospital pharmacy], J Jpn Soc Hosp Pharm, 2010, 46, 149-158.

4) Koinuma M, Miyazaki Y, Okamura M, Okimura A, Sugiura T, Tanaka K, Tahara M, Natsume N, Nakamura H, Ando T, Kase Y, Sato H, Operations Research with Purpose of Promoting Cooperative Instructive Operations at Discharge from Hospital -Activities of Recuperation Sickbed Committee, Japanese Society of Hospital Pharmacists-, Jpn J Pharm Health Care Sci, 2010, 36, 301-309.

5) Japan Pharmaceutical Association, "Heisei 20 Nendo Iryo-Anzen no tameno Yakkyoku-Yakuzaishi to Byoin-Yakuzaishi no Renkei-Suishin Jigyo Soukatsu-Houkokusho" [Annual Report on Communication between Hospital pharmacists and Community Pharmacist for medical safety 2008], Japan Pharmaceutical Association, Tokyo, 2009, pp.5-25.

6) Ministry of Health, Labour and Welfare, Heisei 22 nendo Shinryo-Housyu Kaitei [Revision on remuneration for medical services in fiscal 2010], Ministry of Health, Labour and Welfare Kokuji Dai 59 Gou. 